\title{
Caracterização de crianças e adolescentes com estomas em um serviço de saúde
}

\author{
Characterization of children and teenagers with ostomies in a health service
}

Caracterización de niños y adolescentes con ostomies in a health service

Elaine Carininy Lopes da Costa 1*, Maria Helena Barros de Araújo Luz², Marcia Teles de Oliveira Gouveia², Elaine Maria Leite Rangel Andrade ${ }^{2}$, Paula Cristina Nogueira ${ }^{3}$

ORCID IDS

Costa ECL (D) https://orcid.org/0000-0002-5387-978X

Luz MHBA (iD https://orcid.org/0000-0003-1176-069x

Gouveia MTO (iD https://orcid.org/0000-0002-2401-4947

Andrade EMLR (iD https://orcid.org/0000-0002-1772-7439

Nogueira PC (D) https://orcid.org/0000-0003-4337-2675

\section{COMO CITAR}

Costa ECL, Luz MHBA, Gouveia MTO, Andrade EMLR, Nogueira PC.Caracterização de crianças e adolescentes com estomias em um serviço de saúde. ESTIMA, Braz. J. Enterostomal Ther., 17: e0119. https://doi.org/10.30886/estima.v17.666 PT

Artigo extraído de: Costa ECL. Perfil sociodemográfico e clínico de crianças e adolescentes com estomas [dissertação]. Teresina (PI):Universidade Federal do Piauí, Departamento de Enfermagem; 2016.

\begin{abstract}
RESUMO
Objetivo: Caracterizar o perfil socioeconômico e clínico de crianças e adolescentes com estomias atendidos em um hospital público de referência em saúde infantil do estado do Piauí, Brasil. Métodos: Estudo descritivo, transversal, realizado com 55 crianças/ adolescentes, com pelo menos uma estomia. Resultados: Houve predomínio do sexo masculino, cor parda, média de idade de 4,1 anos. As principais causas para confecção da estomia foram malformações congênitas, destacando-se a anomalia. Com relação ao sistema orgânico, o gastrintestinal foi o mais acometido e a colostomia foi a mais frequente. A maioria dos participantes com estomias de eliminação não utilizava equipamento coletor. Com relação às complicações da estomia, a saída acidental da sonda de gastrostomia foi a mais comum, seguida de sangramento da estomia. A dermatite foi a complicação da pele mais comum. Conclusão: A realização deste estudo possibilitou conhecer o perfil sociodemográfico de crianças e adolescentes com estomia e promover reflexões acerca do cuidado de enfermagem para a melhoria da assistência prestada a essa clientela.
\end{abstract}

DESCRITORES:Estomias cirúrgicas; Criança hospitalizada; Enfermagem; Estomaterapia; Criança; Perfil de saúde.

1.Instituto Federal do Piauí - Departamento de Enfermagem - Oeiras/PI - Brasil.

2.Universidade Federal do Piauí - Departamento de Enfermagem - Teresina/PI - Brasil.

3. Universidade de São Paulo - Escola de Enfermagem de Ribeirão Preto - Ribeirão Preto/SP - Brasil.

Autor correspondente: ninyenfbio@ifpi.edu.br

Recebido: Nov. 21, 2018 | Aceito Jan. 24, 2019 


\begin{abstract}
Objective: To characterize the socioeconomic and clinical profile of children and adolescents with ostomies attended at a public reference hospital in children's health in the state of Piauí, Brazil. Methods: Descriptive cross-sectional study of 55 children/adolescents with at least one ostomy. Results: There was a predominance of males, brown, mean age of 4.1 years. The main causes for the confection of the stoma were congenital malformations, especially the anomaly. Regarding the organic system, the gastrointestinal was the most affected and the colostomy was the most frequent. The majority of participants with elimination ostomies did not use collecting equipment. With regard to the complications of the stoma, the accidental release of the gastrostomy tube was the most common, followed by stomatal bleeding. Dermatitis was the most common skin complication. Conclusion: The realization of this study made it possible to know the socio-demographic profile of children and teenagers with ostomies and to promote reflections about nursing care to improve the assistance provided to these clients.
\end{abstract}

DESCRIPTORS: Surgical ostomies; Hospitalized children; Nursing; Stomatherapy; Kid; Health profile.

\title{
RESUMEN
}

Objetivo: Caracterizar el perfil socioeconómico y clínico de niños y adolescentes con estomias atendidos en un hospital público de referencia en salud infantil del estado de Piauí, Brasil. Métodos: Estudio descriptivo, transversal, realizado con 55 niños/adolescentes, con al menos una estomia. Resultados: Hubo predominio del sexo masculino, color pardo, promedio de edad de 4,1 años. Las principales causas para la confección de la estomia fueron malformaciones congénitas, destacándose la anomalía. Con respecto al sistema orgánico, el gastrointestinal fue el más acometido y la colostomía fue la más frecuente. La mayoría de los participantes con estomias de eliminación no utilizaba equipo colector. Con respecto a las complicaciones de la estomia, la salida accidental de la sonda de gastrostomía fue la más común, seguida de sangrado de la estomia. La dermatitis fue la complicación de la piel más común. Conclusión: La realización de este estudio posibilitó conocer el perfil sociodemográfico de niños y adolescentes con estomia y promover reflexiones acerca del cuidado de enfermería para la mejora de la asistencia prestada a esa clientela.

DESCRIPTORES: Estomias quirúrgicos; Niño hospitalizado; Enfermería; Niño;Estomaterapia; Perfil de salud.

\section{INTRODUÇÃO}

A palavra estomia é de origem grega e significa "boca". Sua confecção é feita por meio de cirurgia, na qual um órgão interno é exteriorizado. Em crianças e adolescentes, as causas mais comuns para a confecção da estomia estão relacionadas à malformação congênita (destacando-se a anomalia anorretal e o mega cólong anglionar congênito), à enterocolite necrotizante e a traumas, podendo ser classificada como temporária (há possibilidade de reconstrução do órgão) ou definitiva (quando não é possível a reconstrução do órgão); o que determinará a possibilidade de reconstrução do órgão é a doença de base e as intervenções cirúrgicas necessárias ${ }^{1,2}$.

No Brasil, não há dados definitivos sobre o número de pessoas com estomias. Segundo o Ministério da Saúde, a cada ano são realizados, aproximadamente, um milhão e 400 mil procedimentos cirúrgicos para confecção de estomias ${ }^{3}$. Dados epidemiológicos sobre o perfil das pessoas com estomia no país, em especial da criança e adolescente, são poucos. O que há, na verdade, são estudos locais ou regionais isolados de caracterização de perfil sociodemográfico e clínico que tratam, em sua maioria, de adultos ou idosos ${ }^{4,5}$.

A escassez de informações oficiais em âmbito nacional e estadual, considerando o perfil da criança e adolescente com estomia, pode prejudicar o planejamento e a implementação de uma política de atenção a essa clientela, bem como dificultar a execução de ações de planejamento no âmbito hospitalar e de gestão para alocação de recursos para a melhoria da assistência para esse grupo específico.

Dessa forma, a realização deste estudo sobre o perfil de crianças e adolescentes com estomias poderá contribuir para a obtenção de dados relevantes sobre o tema, uma vez que possibilitará o dimensionamento das características e necessidades dessa clientela, servindo de subsídio para o direcionamento de políticas de saúde e sociais, bem como para o planejamento de ações para assistência integral às crianças e adolescentes com estomias, melhorando, assim, a qualidade de vida dessas pessoas. Tendo em vista essas considerações, elaborou-se o seguinte objetivo: caracterizar o perfil socioeconômico e clínico de crianças 
e adolescentes com estomias atendidos em um hospital público de referência em saúde infantil em Teresina, estado do Piauí, Brasil.

\section{MÉTODOS}

Trata-se de estudo descritivo do tipo transversal realizado em um hospital público em Teresina, referência na assistência à saúde da criança e adolescente, no período de julho a outubro de 2015. A população foi composta de crianças e adolescentes atendidos nesse centro de assistência. Os participantes foram escolhidos por amostragem de conveniência. Dessa forma, a amostra total foi composta de 55 participantes, sendo 46 crianças e nove adolescentes que atendiam aos seguintes critérios de inclusão: ser criança ou adolescente em tratamento ambulatorial ou internado nesse hospital e apresentar, no momento da coleta de dados, algum tipo de estomia. Foram excluídos da amostra as crianças e adolescentes cujos prontuários, no momento da coleta de dados, não foram disponibilizados pela equipe de saúde.

A identificação das crianças ou adolescentes com estomias era realizada por meio de conversa com a atendente do cirurgião ou com a enfermeira do setor de internação que, de posse dos prontuários, indicava quais eram as crianças e adolescentes com estomia. Após a identificação das crianças e adolescentes, os pais ou responsáveis foram convidados a participar da pesquisa enquanto estavam na sala de espera, aguardando consultas com os profissionais de saúde, ou durante o período de internação da criança ou adolescente. A coleta de dados foi realizada por meio de questionário semiestruturado que investigou as seguintes variáveis:

- Sociodemográficas - Sexo, idade, nível de escolaridade e renda familiar;

- Clínicas - Sistema acometido pela estomia, tipo, formato, coloração, localização anatômica, implantação, diagnóstico médico e causa para confecção, tempo de permanência, existência e tipo de complicação e tipo de equipamento coletor;

- Cirúrgicas - Técnica cirúrgica e momento da cirurgia.

Após os pais ou responsáveis serem informados sobre a realização e os objetivos da pesquisa e terem concordado em participar do estudo, o Termo de Consentimento Livre e Esclarecido foi assinado, responderam ao questionário. $\mathrm{O}$ preenchimento do questionário foi feito pela pesquisadora baseado nas respostas dos pais ou responsáveis pelas crianças ou adolescentes com estomia, na consulta ao prontuário dos participantes do estudo, bem como no exame físico da criança e/ou adolescente, para avaliação das características da estomia.

Os dados foram refinados, tabulados, analisados e estão apresentados em tabelas. Todos os dados oriundos das observações e entrevistas foram transpostos para planilhas eletrônicas do software Microsoft Office Excel® 2010 e posteriormente transportados para o StatisticalPackage for Social Science (SPSS), versão 20.0, para análise estatística descritiva das características sociodemográficas e clínicas das crianças e adolescentes por meio de tabelas de distribuição de frequência absoluta e relativa (porcentagem).

O projeto de pesquisa foi aprovado pelo Comitê de Ética e Pesquisa da Universidade Federal do Piauí por meio do parecer n. 1.115.941 e está de acordo com a Resolução 466/2012 do Conselho Nacional de Saúde referente aos aspectos éticos da pesquisa envolvendo seres humanos. Todos os participantes foram codificados por número de entrevista, não sendo identificadas, em nenhum momento do estudo, suas verdadeiras identidades, sendo preservado o anonimato.

\section{RESULTADOS}

Dos 55 participantes do estudo, 46 (83,6\%) eram crianças e nove $(16,4 \%)$ adolescentes. Trinta e três (60\%) eram do sexo masculino e $32(58,2 \%)$ da cor parda. Quanto à idade, observou-se variação entre a mínima de 10 dias e a máxima de 15 anos e três meses. A faixa etária mais frequente foi a de um a quatro anos, com 20 (36,4\%) crianças; quanto ao nível de escolaridade, $45(81,8 \%)$ não frequentavam creche ou escola. Conforme a renda da família, predominou os que tinham entre um e dois salários mínimos [26 (47,3\%)]. Com relação ao benefício concedido pelo Instituto Nacional de Seguridade Social (INSS) aos portadores de estomias, a maioria 36 $(65,5 \%)$ das crianças e adolescentes não recebia o benefício.

Com relação às crianças, houve predomínio de estomias no sistema gastrointestinal [37 (74\%)]; a colostomia foi a mais frequente [21 (42\%)], seguida 
da gastrostomia [13 (26\%)] e, menos frequentemente, a ileostomia [3 (6\%)] e a pielostomia [1 (2\%)]. Quanto aos adolescentes, predominaram estomias no sistema gastrointestinal [6 (54,7\%)], sendo a gastrostomia a mais frequente $[4(36,6 \%)]$ e, menos frequentemente, a vesicostomia e a cistostomia [1 $(9,1 \%)$ cada uma] (Tabela 1$)$.

Ao considerar as causas básicas para a confecção dos estomias nas crianças, a principal foi a malformação congênita [34 (74\%)] e, nos adolescentes, destacam-se a malformação congênita e a paralisia cerebral [4 $(44,4 \%)$ cada uma]. Com relação aos diagnósticos médicos na criança, a anomalia anorretal foi a mais frequente $[19(34 \%)]$ e o menos comum foi o megacólon congênito [2 (3,5\%)] casos. No adolescente, ocorreu maior frequência do refluxo esofágico [4 (44,3\%)].

Tabela 1. Distribuição das estomias em crianças e adolescentes de acordo com o sistema orgânico acometido e tipo de estomia. Teresina, Piauí, Brasil, $2015(n=55)$.

\begin{tabular}{ccc}
$\begin{array}{c}\text { Sistema } \\
\text { gastrointestinal }\end{array}$ & $\begin{array}{c}\text { Crianças } \\
\mathbf{n}(\%)\end{array}$ & $\begin{array}{c}\text { Adolescente } \\
\mathbf{n}(\%)\end{array}$ \\
\hline Colostomia & $21(42,0)$ & $2(18,1)$ \\
Gastrostomia & $13(26,0)$ & $4(36,6)$ \\
\hline lleostomia & $3(6,0)$ & - \\
\hline Sistema respiratório & Crianças $\mathrm{n}(\%)$ & Adolescente $\mathrm{n}(\%)$ \\
\hline Traqueostomia & $6(12,0)$ & $3(27,1)$ \\
\hline Sistema urinário & Crianças $\mathrm{n}(\%)$ & Adolescente $\mathrm{n}(\%)$ \\
\hline Vesicostomia & $6(12,0)$ & $1(9,1)$ \\
\hline Cistostomia & $-(2,0)$ & $1(9,1)$ \\
\hline Pielostomia & $1(2,0)$ & - \\
Total & $50 *(100,0)$ & $11 *(100,0)$ \\
\hline
\end{tabular}

Fonte: pesquisa direta. ${ }^{*} \mathrm{O}$ número de estomias excede o número de participantes, pois algumas crianças e adolescentes tinham mais de uma estomia.

Tabela 2. Distribuição das crianças e adolescentes com estomia de acordo com as causas básicas e diagnósticos para confecção. Teresina, Piauí, Brasil, $2015\left(n=55^{*}\right)$.

\begin{tabular}{|c|c|c|c|}
\hline \multicolumn{2}{|c|}{ Variáveis } & \multirow{2}{*}{$\begin{array}{c}\text { Crianças n (\%) } \\
34(74,0)\end{array}$} & \multirow{2}{*}{$\begin{array}{c}\text { Adolescentes } \mathrm{n}(\%) \\
4(44,4)\end{array}$} \\
\hline & Malformação congênita & & \\
\hline \multirow[t]{2}{*}{ Causas básicas } & Paralisia cerebral & $7(15,2)$ & $4(44,4)$ \\
\hline & Outras $^{\dagger}$ & $5(10,8)$ & $1(11,2)$ \\
\hline \multirow[t]{4}{*}{ Total } & 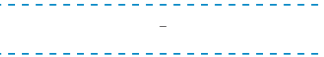 & $46(100,0)$ & $9(100,0)$ \\
\hline & Anomalia anorretal & $19(34,0)$ & $2(22,3)$ \\
\hline & Outros diagnósticos ${ }^{\ddagger}$ & $15(26,8)$ & $2(22,3)$ \\
\hline & Refluxo esofágico & $6(10,7)$ & $4(44,3)$ \\
\hline \multirow[t]{4}{*}{ Diagnósticos médicos } & Atresia de esôfago & $6(10,7)$ & - \\
\hline & Hidronefrose & $5(9,0)$ & $1(11,1)$ \\
\hline & Síndrome de PruneBelly & $3(5,3)$ & - \\
\hline & Megacólon congênito & $2(3,5)$ & - \\
\hline Total & - & $56(100,0)$ & $9(100,0)$ \\
\hline
\end{tabular}

Fonte:pesquisa direta. ${ }^{*}$ O número de estomias excede o número de participantes, pois algumas crianças e adolescentes tinham mais de uma estomia. † Outras causas, como acidente doméstico, infecções e calazar. ${ }^{\ddagger}$ Outros diagnósticos, como insuficiência respiratória, estenose cáustica de esôfago, enterocolitenecrosante, válvula de uretra posterior e estenose uretral.
Destaca-se a ocorrência de outros diagnósticos médicos, enterocolite necrosante, válvula de uretra posterior e estenose uretral (Tabela 2).

Quanto ao formato, predominaram as estomias redondas $[45(73,8 \%)]$ e o menos frequente foi o oval [4 $(6,6 \%)]$. Quanto à cor, a maioria era vermelha muito viva [38 (62,3\%)]. Considerando-se a implantação da estomia na pele, as mais frequentes foram as planas $[33(54,1 \%)] \mathrm{e}$ a menos frequentes foram as retraídas [2(3,3\%)]. No que diz respeito à localização da estomia, predominaram as do quadrante inferior esquerdo [23 (36,5\%)] seguidas das da região epigástrica [17 (27\%)]. Quanto à técnica cirúrgica para confecção dos estomias intestinais, houve predomínio da técnica em duas bocas $[33(53,8 \%)]$ seguida da técnica em alça [23 $(38,5 \%)]$. No que diz respeito ao caráter da cirurgia, $33(60 \%)$ a realizaram em caráter de urgência e 22 (40\%) a fizeram de forma programada.

Com relação ao uso do equipamento coletor de efluente pelas 34 crianças e adolescentes com estomias de eliminação, constatou-se que apenas sete (20,6\%) utilizavam tal equipamento. Quanto aos tipos de dispositivos utilizados para alimentação dos participantes que tinham gastrostomia, verificou-se que a grande maioria [13 (76,6\%)] utilizava a cateter de Foley e apenas quatro $(23,5 \%)$ o dispositivo de baixo perfil do tipo válvula de Mic-Key.

No que se refere às complicações na estomia, apenas as crianças $[8(17,4 \%)]$ as apresentaram, sendo que em quatro (50\%) ocorreu a saída acidental do cateter de Foley da gastrostomia, o que constitui em complicação do como insuficiência respiratória, estenose cáustica do esôfago, 
dispositivo, três $(37,5 \%)$ tiveram sangramento e uma $(12,5 \%)$ prolapso na ileostomia. Ao considerar a pele periestomal, $13(23,6 \%)$ apresentaram complicações, sendo 11 crianças e dois adolescentes, com predomínio da dermatite irritativa [12 (92,3\%)] (Tabela 3).

Tabela 3. Distribuição das crianças e adolescentes com estomia que apresentaram complicações das estomias e da pele periestoma. Teresina, Piauí, Brasil, 2015 ( $n=55)$.

\begin{tabular}{|c|c|c|}
\hline \multicolumn{2}{|c|}{ Variáveis } & n (\%) \\
\hline \multirow{3}{*}{$\begin{array}{l}\text { Complicação da } \\
\text { estomia } \\
(\mathrm{n}=8)\end{array}$} & $\begin{array}{c}\text { Saída da sonda } \\
\text { gastrostomia }\end{array}$ & $4(50,0)$ \\
\hline & Sangramento & $3(37,5)$ \\
\hline & Prolapso & $1(12,5)$ \\
\hline Complicações na pele & Dermatite irritativa & $12(92,3)$ \\
\hline periestoma ( $n=13$ ) & Pústula & $1(7,7)$ \\
\hline
\end{tabular}

Fonte: pesquisa direta.

\section{DISCUSSÃO}

Este estudo evidenciou que a maioria das crianças e adolescentes com estomia era do sexo masculino, o que coincide com os resultados encontrados em publicações da Colômbia, Nigéria, África do Sul, Coreia do Sul e Brasil ${ }^{6-14}$. Em contrapartida, há um estudo brasileiro com predomínio de crianças e adolescentes do sexo feminino com estomia ${ }^{15}$. Com relação à cor da pele, a maioria dos participantes referiu a cor parda, o que reflete as características próprias da Região Nordeste, diferindo dos resultados de um estudo realizado em São Paulo, em que houve predomínio da cor branca $^{15}$.

Ao considerar a idade dos participantes, observou-se diversidade de crianças desde o período neonatal, escolar, pré-escolar e início da adolescência, que variou entre a mínima de 10 dias a 15 anos e 3 meses. Esse resultado aproxima-se de um estudo com crianças com estomia no qual houve predomínio de crianças na faixa etária de um a quatro anos ${ }^{6,13,14}$.

Quanto às doenças do trato gastrointestinal que resultam na confecção de estomias, a literatura mostra que há variação na faixa etária entre crianças e adolescentes, sendo comum a ocorrência desde os primeiros dias de vida até a adolescência, porém ocorre com maior predomínio na faixa etária entre zero e cinco anos ${ }^{9,16,17}$.

Com relação à renda familiar, houve predomínio de pessoas com renda familiar entre um e dois salários mínimos (47,3\%). Esses resultados coincidem com os de outras pesquisas de perfil de pessoas com estomia em que predominava a renda familiar abaixo de três salários mínimos ${ }^{2,5,12,14}$. Também foram identificadas famílias de crianças e/ou adolescentes com renda de até um salário mínimo (34,5\%). Corroborando esse achado, pesquisas com crianças e adolescentes com estomia evidenciaram famílias com renda per capta menor que um salário mínimo ${ }^{12,17}$. A informação da renda permite concluir que a população pesquisada tem baixo poder aquisitivo, o que dificulta a aquisição de dispositivos coletores adequados ao tipo de estomia, bem como dos acessórios adjuvantes.

Com relação ao nível de escolaridade, verificou-se que a maioria não frequentava a escola $(81,8 \%)$. Tal resultado está relacionado à idade dos participantes, pois $27,2 \%$ eram menores de um ano, ou seja, ainda não estavam em idade escolar, o que coincide com um estudo realizado em Belém ${ }^{14}$. Outro motivo apontado pelos pais diz respeito à insegurança em colocar o filho com estomia na escola, pois, segundo eles, essas não tinham professores preparados para tal clientela. Além disso, tinham medo da reação dos colegas, o que influenciava na decisão de não matricular os filhos.

Esses resultados assemelham-se aos encontrados em um estudo com crianças com estomia em que $74,4 \%$ dos entrevistados não frequentavam a escola, sendo os motivos a idade da criança (60\% de lactentes), a situação de saúde da criança que impossibilitava frequentar a escola (problemas neurológicos), a distância entre a escola e a residência, o temor dos pais de vazamento de efluente e, consequentemente, a necessidade de esvaziamento ou troca da bolsa, expondo assim a criança ${ }^{6}$.

Ao considerar os sistemas acometidos pelas estomias nos participantes da pesquisa, houve predomínio de estomias no sistema gastrointestinal (68\%). Esse resultado é semelhante ao de outras pesquisas em que esse foi o mais acometido ${ }^{13,14,18-21}$. Apesar de a literatura evidenciar que o sistema gastrointestinal é o mais acometido por estomias em crianças e adolescentes, foi possível encontrar pesquisas em que o sistema orgânico mais acometido foi o urinário ${ }^{15}$.

Com relação ao tipo de estomia, a colostomia foi a mais frequente. Esses resultados assemelham-se a outros estudos com crianças com estomia em que houve maior ocorrência da colostomia ${ }^{10,12-14,17-24}$. Destaca-se, na literatura, a existência de outros estudos em pediatria que evidenciam o predomínio de urostomia, gastrostomia e até mesmo a ileostomia ${ }^{10,14}$.

No que se refere às causas básicas para confecção das estomias em crianças e adolescentes, predominaram 
as malformações congênitas. Dessa forma, os resultados encontrados neste estudo assemelham-se aos outros de predomínio das malformações congênitas ${ }^{8,10,12,17,21}$. Considerando as estomias intestinais, houve maior frequência de anomalia anorretal. Alguns estudos com crianças com estomia corroboram esses resultados ${ }^{12,19}$.

Em contrapartida, apesar da pequena quantidade de casos de megacólon congênito encontrado neste estudo, pôde-se perceber, na literatura, que a frequência desse diagnóstico médico para confecção de estomias em crianças foi igual ao de anomalia anorretal e, em alguns estudos, o

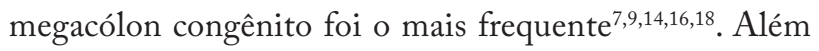
das causas citadas, foi possível encontrar estudos em que a causa principal para confecção da estomia foi o trauma com perfuração abdominal por arma de fogo ${ }^{22}$.

No que diz respeito ao formato da estomia, houve predomínio do formato redondo $(73,8 \%)$ e em relação à cor e predominaram estomias com a coloração vermelha muito viva. É importante destacar que as características normais de uma estomia são: coloração rosa forte ou vermelha muito viva, formato regular, no qual o corpo da estomia apresenta a mesma forma de sua base na parede abdominal, brilhante e geralmente com presença de muco, pele íntegra e sem alterações (edema, sangramento, ulceração ou nódulos em sua mucosa $)^{13,24}$. Considerando a localização das estomias intestinais, predominaram as localizadas no quadrante inferior esquerdo. Corroborando esses resultados, pesquisas apontam o predomínio de estomias intestinais localizadas no quadrante inferior esquerdo ${ }^{24}$.

Quanto à técnica cirúrgica para confecção dos estomias intestinais, houve predomínio da técnica em duas bocas. Em concordância com esses achados, foi possível encontrar pesquisas com maior frequência de estomias intestinais realizadas em técnica de duas bocas ${ }^{13,21}$. $\mathrm{Na}$ técnica de duas bocas, ocorre a abertura do segmento intestinal onde as duas bocas ficam unidas, sendo uma boca proximal, por onde sai o efluente, e outra distal, que não funciona e que se comunica com o reto e o ânus.

No que se refere ao caráter da cirurgia, a maioria a realizou em caráter de urgência. As cirurgias para construção de estomias em neonatos são feitas de emergência, geralmente poucos dias após o nascimento, sendo incomum a demarcação do local da estomia. Muitas vezes a família não tem poder de escolha, pois a demora na realização da cirurgia pode implicar em piora do estado de saúde da criança para situações irreversíveis e até mesmo a morte ${ }^{24}$.
É importante destacar que, nos casos de cirurgias eletivas, a assistência de enfermagem deve ser realizada de forma eficaz, iniciando no pré-operatório por meio da consulta de enfermagem e da demarcação do local da estomia pelo enfermeiro estomaterapeuta ou enfermeiro com treinamento especializado; dessa forma, a maioria das complicações com a estomia e a pele periestoma poderia ser possivelmente evitada ${ }^{24}$.

Evidenciaram-se, neste estudo, 34 participantes com estomias de eliminação em que a maioria $(79,4 \%)$ não utilizava equipamento coletor de efluente, sendo esse substituído por fraldas de pano, e sete faziam uso do equipamento coletor de fezes ou urina. Tal resultado também foi encontrado em outras pesquisas realizadas com crianças com estomia, em que os pais ou cuidadores familiares não utilizavam equipamento coletor, e sim fralda de pano ou gaze fixada com atadura para contenção do efluente ${ }^{2,16,18,23}$. O uso do equipamento coletor em pessoas com estomias de eliminação é um direito assegurado pela legislação e que possibilita melhor qualidade de vida e redução de dermatites.

Ao investigar os motivos para o não uso do equipamento coletor, destaca-se a dificuldade dos pais para adquirir as bolsas, principalmente de ordem econômica, pois a maioria tem renda de até dois salários mínimos, o que dificulta a compra do equipamento. Além disso, este estudo revelou que alguns pais desconheciam o programa de distribuição de bolsas coletoras realizado em Teresina, o que pode demonstrar a orientação incipiente aos pais sobre o programa pelos profissionais envolvidos com a assistência.

Corroborando esses achados, uma pesquisa sobre as dificuldades relatadas por familiares cuidadores de crianças com estomia destacou a presença de familiares que não receberam nenhum tipo de orientação sobre o polo de distribuição dos equipamentos coletores, além de casos em que o familiar só teve conhecimento da existência do polo após um ano da confecção da estomia e que durante todo esse período eram utilizadas fraldas de pano para contenção dos efluentes ${ }^{23}$.

Outro motivo apontado pelos pais diz respeito à falta de conhecimento sobre a troca e a higiene da bolsa coletora. Essa informação é relevante, uma vez que mostra a fragilidade da assistência de enfermagem não só às crianças, mas também aos seus familiares, pois o cuidado com a criança com estomia exige, da família, a aquisição de novas competências e habilidades que não fazem parte 
do seu cotidiano. Dessa forma, necessitam de apoio dos profissionais envolvidos com a assistência, em especial da enfermagem.

Com relação aos dispositivos utilizados para a alimentação dos participantes com gastrostomia, observou-se que a maioria [13 (76,6\%)] utilizava o cateter de Folley para alimentação. É importante destacar, mais uma vez, que a população estudada tem baixa renda, o que justifica a dificuldade na aquisição de dispositivos de baixo perfil, como a válvula de Mic-Key ${ }^{\oplus}$. Dessa forma, os participantes dependem quase exclusivamente dos dispositivos oferecidos no hospital de confecção da gastrostomia, que na maioria das vezes disponibiliza o cateter do tipo Folley por ser mais econômico.

No que diz respeito às complicações relacionadas às estomias,observou-se que, do total, apenas oito crianças apresentaram complicações com a estomia, sendo mais frequente a saída acidental da sonda da gastrostomia seguida do sangramento na estomia e, o menos frequente, o prolapso da estomia. Tal resultado corrobora os resultados de um estudo que também encontrou, como complicação mais frequente, a saída acidental do cateter de alimentação ${ }^{25}$. Em contrapartida, diferentemente do que foi encontrado neste estudo, ao buscar na literatura as principais complicações de estomias intestinais em crianças e adolescentes, identificou-se, em estudos internacionais, que o prolapso da estomia foi a principal complicação encontrada ${ }^{11,21}$.

Em pesquisa realizada no Brasil, evidenciou-se a ocorrência de complicações em 80\% das crianças estudadas, sendo as mais frequentes o sangramento no local da estomia e a hérnia periestomal ${ }^{16}$. Neste estudo, $24 \%$ dos participantes apresentaram complicações, sendo que a dermatite irritativa foi a mais prevalente $(92,3 \%)$. Tal ocorrência corrobora o resultado de estudos que relataram a grande frequência da dermatite irritativa, geralmente associada ao manuseio inadequado do equipamento coletor, bem como o contato do efluente liberado pela estomia, com especial atenção à ileostomia, na qual o efluente alcalino aumenta os riscos para desenvolvimento de tais lesões ${ }^{4,5,11}$. Em contrapartida, também foi possível encontrar, na literatura, pesquisa apontando a menor frequência de dermatite periestoma ${ }^{12}$.

Ressalta-se que a presença da estomia de eliminação exige, de toda equipe de saúde, em especial do enfermeiro, um cuidado efetivo na promoção da saúde e na prevenção de agravos, tais como as complicações na estomia e na pele periestomal ${ }^{12}$. Nessa perspectiva, o enfermeiro deve orientar os pais e/ou familiares acerca do cuidado à criança ou adolescente como um todo, tendo em vista que a confecção da estomia pode acarretar transtornos nas diversas dimensões da vida da criança e da família. Além disso, é importante que, para possibilitar a inserção e o sucesso dessa criança ou adolescente na escola, o professor também seja parceiro e receba, assim como os pais, orientações sobre a higienização das estomias, a colocação e retirada correta, a identificação dos aspectos relacionados a normalidades e manifestações precoces de alterações, tornando-os capacitados a desenvolver ações preventivas e de reabilitação.

É importante destacar que, além dos cuidados com a estomia de eliminação, a equipe de enfermagem também deve orientar os pais ou cuidadores sobre os cuidados com a gastrostomia, e dentre eles destaca-se: a limpeza com solução fisiológica 0,9\%, a manutenção da pele periestomal limpa e seca, a lavagem do equipamento de alimentação, o uso de protetor cutâneo spray ou hidrocoloide ao redor da estomia, bem como as informações sobre sinais de complicação e infecção da gastrostomia. Outra recomendação importante diz respeito aos casos de saída acidental do cateter. Nessa situação, o paciente e/ou familiar deverá posicioná-lo provisoriamente na gastrostomia e procurar assistência médica o mais rápido possível para sua recolocação, devido ao risco de fechamento da estomia caso esse período ultrapasse 24 horas $^{26}$.

Ressalta-se que não basta o técnico procedimental; é fundamental o acolhimento, a escuta qualificada, a humanização do cuidado e as orientações acerca dos direitos da pessoa com estomia, dentre eles a aquisição gratuita de equipamentos coletores. Além disso, destaca-se que, sempre que possível, a criança e o adolescente sejam ouvidos e incluídos no planejamento das ações para o cuidado e o autocuidado.

\section{CONCLUSÃO}

Com o desenvolvimento deste estudo, foi possível traçar o perfil socioeconômico e clínico de crianças e adolescentes com estomias que se encontravam em tratamento ambulatorial ou na unidade de internação de um hospital público de referência em saúde infantil do estado do Piauí, evidenciando o predomínio de participantes do sexo masculino, cor parda, na faixa etária de um a quatro anos, que ainda não frequentavam escola, sendo a renda familiar entre um e dois salários mínimos. Com relação ao tipo de estomias, a 
colostomia foi a mais frequente e a malformação congênita foi a principal causa para sua confecção.

O estudo também evidenciou crianças com estomias de eliminação que não faziam uso de equipamento coletor de efluente e sim fralda de pano, sendo os motivos apontados para tal a falta de conhecimento dos pais em relação ao manuseio do equipamento coletor e a forma de aquisição desse equipamento, o que pode estar relacionado à orientação incipiente oferecida pelos profissionais de saúde, especialmente do enfermeiro e de sua equipe. É importante destacar que a maioria das estomias confeccionadas foi realizada em caráter de urgência, o que impossibilitou sua demarcação prévia, o que pode favorecer o aparecimento de complicações, tais como a dermatite periestomal.

O estudo poderá contribuir para os profissionais de saúde, especialmente o enfermeiro, para promover reflexões acerca de sua prática assistencial e do planejamento de suas ações fundamentadas na legislação em vigor e nos direitos da pessoa com estomia, tais como, ter um estomia bem demarcada, receber orientações sobre os cuidados e aquisição gratuita de equipamentos coletores e adjuvantes. Nessa perspectiva, tal reflexão contribuirá para o melhor planejamento da assistência a essa clientela e aos seus pais e/ou familiares, resultando, dessa forma, em melhoria na qualidade do cuidado prestado.
Embora a maioria dos participantes deste estudo não frequentasse a escola $(81,8 \%)$ por não estar em idade escolar, chama atenção a insegurança dos pais em matricular o filho com estomia, ao alegarem receio quanto ao despreparo dos professores no cuidado da criança e da reação dos colegas. É premente que os professores estejam preparados para receber e incluir a criança e o adolescente na escola e para tal os enfermeiros estomaterapeutas podem e devem prepará-los oferecendo apoio, informações claras, fôlderes e capacitação.

As principais limitações dessa pesquisa foram: delineamento do estudo, realização em única instituição e a escassez de publicações que contemplassem os aspectos morfológicos dos estomias em crianças e adolescentes.

\section{CONTRIBUIÇÃO DOS AUTORES}

Conceitualização,Costa ECL;Luz MHBA;Gouveia MTO; Andrade EMLR e Nogueira PC; Metodologia,Costa ECL; Investigação, Costa ECL; Redação - Primeira versão: Costa ECL;Luz MHBA;Gouveia MTO; Andrade EMLR e Nogueira PC; Redação Revisão \& Edição,Costa ECL;Luz MHBA;Gouveia MTO; Andrade EMLR e Nogueira PC; Aquisição de Financiamento, CostaECL; Recursos, Costa ECL; Supervisão, Costa ECL.

\section{REFERÊNCIAS}

1. Coldicutt P, Hill B. An overview of surgical stoma construction and its effects on the child and their family. Nurs Child Young People. 2013;25(4):26-34.

2. Poleto D, Gonçalves MI, Barros MTT, Anders JC, Martins ML. A criança com estoma intestinal e sua família: implicações para o cuidado de enfermagem. TextoContextoEnferm. 2011;20(2):319-27.

3. LenzaNFB, Sonobe HM, BuettoLS, Santos MG, Lima MS. The teaching of self-care to ostomy patients and their families: an integrative review. Rev Bras Promoç Saúde Fortaleza;26(1):139-45.https://doi.org/10.5020/2644

4. Luz ALA, Luz MHBA. Caracterização de pessoas com estomias intestinais de eliminação na saúde da família: contribuição para a enfermagem. ESTIMA, Braz J Enterostomal Ther. 2014;12(2):35-5.

5. Luz ALA, Luz MHBA, Antunes A, Oliveira GS, Andrade EMLR, Miranda SM. Perfil de pacientes estomizados: revisão integrativa da literatura. Cult Cuid. 2014;18(1): 115-23.

6. Dias SM, Gonçalves FG. Percepções de familiares com criança estomizada sobre o grupo de apoio. ESTIMA, Braz J Enterostomal Ther. 2015;13(2):70-5.
7. Almeida EJ, Silva AL. Caracterização do perfil epidemiológico dos estomizados em hospitais da Secretaria de Estado de Saúde do Distrito Federal. ESTIMA, Braz J Enterostomal Ther. 2015;13(1):1-16. https://doi.org/10.5327/Z18063144201500010004

8. Rodrigues LN, Silva AMO, Xavier MS, Chaves EMC. Complicações e cuidados relacionados ao uso da gastrostomia em pediatria. ESTIMA, Braz J Enterostomal Ther. 2018;16(e1018):1-6. https://doi.org/10.30886/estima. v16.464 PT

9. Gamboa NSG, Álvarez LST. Cuidado de un hijo ostomizado: cambios en la familia. Avenferm. 2013;31(1):59-71.

10. Monteiro SNC, Kamada I, Silva AL, Souza TC. Perfil de crianças e adolescentes estomizados atendidos de um hospital público do Distrito Federal. ESTIMA, Braz J Enterostomal Ther. 2014;12(3):23-32.

11. Nam SH, Kim DY, Kim SC. Can we expect a favorable outcome after surgical treatment for an anorectal malformation? J Pediatr Surg. 2016;51(1):421-4. https://doi. org/10.1016/j.jpedsurg.2015.08.048.

12. Monteiro SNC, Kamada I, Silva AL. Qualidade de vida: percepção de crianças e adolescentes estomizados e 
seus pais e/ou responsáveis. ESTIMA, Braz J Enterostomal Ther. 2016;14(1):50. https://doi.org/10.5327/Z1806-31442 $\underline{01600010008}$

13. Costa ECL, Vale DS, Luz MHBA. Perfil das crianças estomizadas em um hospital público de Teresina, Piauí. ESTIMA, Braz J Enterostomal Ther. 2016;14(4):169-74. https://doi.org/10.5327/Z1806-3144201600040003

14. Bezerra PD, Pinto ISM, Cunha RR, Ramos EMLS, Silva CO, Ferreira SRM. Perfil sociodemográfico e clínico de crianças com estomias em um centro de referência, Belém (PA) ESTIMA, Braz J Enterostomal Ther. 2017;15(4):214-21. https://doi.org/10.5327/Z1806-3144201700040005

15. Melo MC, Kamada I. O papel da família no cuidado à criança com estoma intestinal: uma revisão narrativa. ESTIMA, Braz J Enterostomal Ther. 2015;13(3):121-6.

16. Rosado SR, DázioEMR, Siepiersk CT, Filipini CB, Fava SMCL. O cuidado de enfermagem e as lacunas na assistência à criança com estomias: uma revisão integrativa. ESTIMA, Braz J Enterostomal Ther. 2015;13(2):83-7.

17. Egito ETBN, Medeiros AQ, Moraes MM, Barbosa JM. Estado nutricional de pacientes pediátricos ostomizados. Rev Pau Pediatr. 2013;31(1):58-64

18. Menezes HF, GoésFG, Maia SM, Souza AL. A subjetividade no cuidado familiar à criança ostomizada a partir da construção da sua autonomia. RevPesquiCuid Fundam. 2016;5(2):37319. https://doi.org/10.9789/2175-5361.2013v5n2p3731

19. Rosado SR, DázioEMR, Fava SMCL, Maia PH, Filipini CB, ReskZMR. Experiência de ser mãe de criança com estomia. ESTIMA, Braz J Enterostomal Ther. 2014;12(1):12-20.

20. Santos OJ, Filho ENS, Filho AKDB, Desterro VS, Silva MVT, Prado RPS, et al. Children and adolescents ostomized in a reference hospital. Epidemiological profile. J Coloproctol. 2016; 36(2):75-9. https://doi.org/10.1016/j. jcol.2016.03.005.

21. ZacarinCFL, Alvarenga WA, Souza ROD, Borges DCS, Dupas G. Vulnerabilidade da família de crianças com estomia intestinal. RevEletr Enf. 2014;16(2):426-33. https://doi. org/10.5216/ree.v16i2.26639

22. Oda O, Davies D, Colapinto K, Gerstle T. Loop versus divided colostomy for the management of anorectal malformations. J Pediatr Surg. 2014;49(1):87-90. https:// doi.org/10.1016/j.jpedsurg.2013.09.032

23. Liechty ST, Barnhart DC, Huber JT, Zobell S, Rollins MD. The morbidity of a divided stoma compared to a loop colostomy in patients with anorectal malformation. J PediatrSurg. 2016;51(1):107-10. https://doi.org/https://doi. org/10.1016/j.jpedsurg.2015.10.025

24. SpenazatoRVS, Santos CRS, MaritaABPS, Paula MAB. Satisfação de clientes com estomias intestinais quanto ao uso de equipamentos. ESTIMA, Braz J Enterostomal Ther. 2016;14(4):193-202. https://doi.org/10.5327/Z18063144201600040006

25. Rodrigues LN, Silva AMO, Xavier MS, Chaves EMC. Complicações e cuidados relacionados ao uso do tubo de gastrostomia em pediatria ESTIMA, Braz J Enterostomal Ther. 2018;16:e1018. https://doi.org/10.30886/estima. v16.464_PT.

26. Silva TP, Ribeiro CRG, ResckZMR, Fava SMCL, DázioEMR. Cuidado de enfermagem à pessoa com gastrostomia: revisão integrativa. ESTIMA, Braz J Enterostomal Ther. 2018;16: e0718. https://doi.org/10.30886/estima.v16.374_PT 